\title{
Charta «Zusammenarbeit der Fachleute im Gesundheitswesen»
}

Schweizerische Akademie der Medizinischen Wissenschaften (SAMW)

Eine Stellungnahme der FMH findet sich im Anschluss an diesen Beitrag auf Seite 1806.
Korrespondenz:

Schweizerische Akademie der Medizinischen Wissenschaften (SAMW)

Petersplatz 13

CH-4051 Basel

mail[at]samw.ch

\section{Präambel}

Die Schweizerische Akademie der Medizinischen Wissenschaften (SAMW) setzt sich seit 1999 unter dem Titel «Zukunft Medizin Schweiz» intensiv mit den Zielen und Aufgaben der Medizin auseinander. Im Rahmen dieses Projektes veröffentlichte eine Arbeitsgruppe 2007 den Bericht «Zukünftige Berufsbilder von Ärztinnen, Ärzten und Pflegenden in der ambulanten und klinischen Praxis». Eine der Empfehlungen dieses Berichts betraf die Ausarbeitung einer «Charta» zur Zusammenarbeit der Gesundheitsfachleute. Expertengespräche haben bestätigt, dass eine solche Charta sinnvoll ist und einen Beitrag dazu leisten könnte, die aktuellen und kommenden Herausforderungen des Gesundheitswesens zu bewältigen.

Die vorliegende Charta soll dazu beitragen, a) die Behandlung der Patienten zu optimieren, b) angesichts des zunehmenden Mangels an Gesundheitsfachleuten die Versorgung sicherzustellen und c) die Arbeit der Gesundheitsfachleute zu unterstützen. Die Charta nimmt dabei Bezug auf ähnliche Initiativen im Ausland.

Mit der Charta richtet sich die SAMW primär an die in der Gesundheitsversorgung aktiven Fachleute, Berufsverbände und Institutionen; sie soll Ausdruck sein für den Geist der Zusammenarbeit der Gesundheitsfachleute und eine Basis für den anzustrebenden Kulturwandel. Gleichzeitig möchte sie die zahlreichen Initiativen im Gesundheitswesen, die auf eine Zusammenarbeit der Gesundheitsfachleute angewiesen sind (u. a. Strategie Palliative Care, Strategie Demenz, Strategie gegen Krebs), unterstützen.

Für eine fruchtbare und erfolgreiche Zusammenarbeit der Gesundheitsberufe braucht es auch geeignete Rahmenbedingungen; entsprechend sind auch Politik, Verwaltung und Versicherer eingeladen, auf die Charta Bezug zu nehmen.

Die Charta ist ein dynamisches Dokument; sie kann und wird sich entwickeln und verändern. Die Adressaten sind eingeladen, diesen Entwicklungsund Veränderungsprozess als Chance zu betrachten und aktiv dazu beizutragen.

\section{Prinzipien}

Die Angehörigen aller Gesundheitsberufe setzen sich für eine bedarfs- und bedürfnisorientierte, qualitativ hochstehende Gesundheitsversorgung der Bevölkerung ein.
Im Zentrum der Gesundheitsversorgung stehen die Patientin bzw. der Patient. Dies bedeutet, dass die edukativen, beratenden, präventiven, diagnostischen, therapeutischen, pflegerischen, rehabilitativen und palliativen Leistungen aller benötigten Berufsleute aufeinander abzustimmen sind. Die Verantwortung wird entsprechend den fachlichen Kompetenzen wahrgenommen. Die gegenseitige Information ist jederzeit gewährleistet, die Arbeit basiert auf gegenseitigem Respekt und gemeinsam definierten, anerkannten Standards.

Die Ziele einer solchen Gesundheitsversorgung sind der gleichberechtigte Zugang der Bevölkerung zu Gesundheitsleistungen, dies in guter Qualität, mit hoher Patienten- und Mitarbeiterzufriedenheit sowie Effizienz. Sie ist damit Teil einer «nachhaltigen Medizin», die sich an den Kriterien der Evidenz, der Ethik und der Ökonomie orientiert.

\section{Kernelemente und Verpflichtungen}

\section{Die interprofessionelle Zusammenarbeit bindet Patienten als Partner ein.}

Informierte Patientinnen und Patienten und ihre Angehörigen wollen zunehmend eine aktive Rolle im Gesundheitswesen einnehmen und sich an Entscheidungen zur Therapie und Versorgung beteiligen. Sie sind als «Experten in eigener Sache» massgeblich für die Behandlungsqualität und das Ergebnis mitverantwortlich.

\section{Die Gesundheitsberufe klären für konkrete Arbeitssituationen in einem gemeinsamen Prozess ihre Kompetenzen und Verantwortlich- keiten und halten diese fest; bei überlappenden Kompetenzen sind die Verantwortlichkeiten explizit zu bezeichnen.}

Für einen Teil dieser Klärungsprozesse können allgemein akzeptierte Definitionen und Richtlinien erarbeitet werden, der andere Teil ist sinnvollerweise jeweils innerhalb der einzelnen Institutionen bzw. Organisationen festzulegen (Spital, Ambulatorium, Gesundheitszentrum, Praxisgemeinschaft, Netzwerk, Spitexorganisation, stationäre Langzeitpflege u.a.). Für die Übernahme von zusätzlicher Verantwortung braucht es einen klar definierten Rahmen (z.B. im Falle von erweiterten Diagnose-, Verordnungsund Abrechnungskompetenzen für die beteiligten Gesundheitsberufe). 
3. Die Übernahme von Aufgaben im Versorgungsprozess orientiert sich am Bedarf der Patienten und ihrer Angehörigen und an den benötigten fachlichen Kompetenzen.

Die Aufgabenverteilung orientiert sich nicht an hierarchischen Kriterien und/oder an traditionellen Berufsbildern. Die Berufsgruppen sind bereit, je nach Situation Aufgaben abzugeben oder Verantwortung neu zu übernehmen. Dies bedeutet, dass die Gesundheitsfachleute entsprechend ihren spezifischen Kompetenzen und Fähigkeiten tätig sind und für dieses Handeln auch Verantwortung tragen.

4. Die Berufs- und Fachverbände entwickeln unter Berücksichtigung internationaler Empfehlungen - gemeinsame, interprofessionelle Leitlinien; auf institutioneller Ebene ergänzen die verschiedenen Berufsgruppen diese mit gemeinsamen, interprofessionellen Standards.

Diese Leitlinien und institutionellen Standards bilden Leitplanken; deren Beachtung schafft gegenseitiges Vertrauen. Ein Abweichen von Leitlinien und Standards ist möglich, bedarf jedoch der Begründung.

\section{Sowohl für die Ausbildung als auch für die} Weiterbildung gibt es gemeinsame Module. Die Aus- und Weiterbildungsstätten der verschiedenen Gesundheitsberufe sind vernetzt.

Im Hinblick auf die interprofessionelle Berufspraxis umfassen die Aus- und Weiterbildung der Gesundheitsberufe die notwendigen berufsübergreifenden und kooperativen Elemente. Die Ausbildung vermittelt im Rahmen von gemeinsamen Modulen allen Gesundheitsberufen neben ihren jeweiligen spezifischen Kenntnissen gemeinsame Grundelemente
(z.B. Basiswissen über Gesundheit und Krankheit sowie neben Kommunikation, Ethik, Gesundheitsökonomie und -politik auch die Prinzipien der interprofessionellen Kooperation).

6. Das lebenslange Lernen und damit verbunden der Nachweis früher und neu erworbener Kompetenzen und Fertigkeiten sind für alle Berufs- und Fachverbände eine Selbstverständlichkeit und werden durch Arbeitgeber/Fachverbände gefördert.

Das Wissen und das Spektrum der Tätigkeiten bei Einstieg in den Beruf sind nicht mehr identisch mit denjenigen zum Zeitpunkt der Pensionierung. Lebenslange, gezielte Fortbildung, der Erwerb neuer Kompetenzen und die Bereitschaft zur stetigen Sicherung der Qualität im eigenen Tätigkeitsfeld sind Voraussetzungen zur Ausübung der Berufe im Gesundheitswesen. Geeignete Fortbildungsangebote sind für alle in Frage kommenden Berufsgruppen zugänglich und werden - falls sinnvoll - von Vertretern verschiedener Berufsgruppen gemeinsam organisiert.

7. Die Arbeitsmodelle sind so ausgerichtet, dass sie eine integrierte Betreuung fördern und unterstützen.

Gesundheitseinrichtungen mit Zentrumsfunktion, bei denen alle in einen Versorgungsprozess involvierten Berufsgruppen angestellt sind und koordiniert werden, bieten gute Voraussetzungen für ein kompetenzorientiertes Miteinander und dienen als Drehscheibe für die regionale Versorgung; weitere Partner in der Region können durch ein Netzwerk eingebunden werden. Um den gegenseitigen, kontinuierlichen Informationsaustausch sicherzustellen,

Hinweise zur Ausarbeitung dieser Charta

Der Arbeitsgruppe, die diese Charta ausgearbeitet hat, gehörten folgende Persönlichkeiten an:

Dr. Werner Bauer (Vorsitz), Präsident SIWF, Küsnacht ZH; Dr. Hermann Amstad, Generalsekretär SAMW, Basel; Marie-Anne Becker, Hôpital Neuchâtelois, Neuchâtel; Dr. Manuela Eicher, Haute Ecole de Santé HES-SO, Fribourg; Prof. Viola Heinzelmann, Universitäts-Frauenspital, Basel; Dr. Isabelle Meier, mediX Gruppenpraxis, Zürich; Maja Mylaeus-Renggli, Spitex Verband Schweiz, Bern; Dr. Hans Neuenschwander, IOSI/EOC, Bellinzona; Prof. Cornelia Oertle, Berner Fachhochschule Gesundheit, Bern; Dr Pierre-Yves Rodondi, Spécialiste en Médecine interne générale, Pully; Martine Ruggli, pharmaSuisse, Liebefeld; Prof. Astrid Schämann, ZHAW, Winterthur; Dr. Beat Sottas, Careum, Zürich; Prof. Peter M. Suter, Vizepräsident SAMW, Presinge.

Die Arbeitsgruppe hat sich ab April 2013 in mehreren Sitzungen vertieft mit der Thematik auseinandergesetzt. Nach Sichtung der Literatur und eingehenden Diskussionen hat sie einen ersten Entwurf der Charta verfasst; diesen hat sie Anfang Oktober 2013 in einem Hearing von ausgewählten Expertinnen und Experten kommentieren lassen (Dr François Héritier, Präsident SGAM, Courfaivre; Roland Paillex, Präsident physioswiss, Lausanne; Pierre Théraulax, Präsident SBK-ASI, Bern; lic. oec. Rita Ziegler, Direktionsvorsitzende UniversitätsSpital Zürich, Dr. Alan Niederer, Wissenschaftsjournalist, Zürich; lic. rer. pol. Michael Jordi, Zentralsekretär GDK, Bern). Im Anschluss daran wurde ein überarbeiteter Entwurf einer breiten öffentlichen Vernehmlassung unterzogen. Die Mehrheit der Stellungnahmen begrüsste zwar die Ausarbeitung einer Charta, doch war einerseits in Ärztekreisen eine grössere Skepsis spürbar und wünschten andererseits zahlreiche Institutionen, dass sie stärker in den Ausarbeitungsprozess eingebunden würden. Auch die Umsetzungsvorschläge wurden eher kritisch gesehen. Vor diesem Hintergrund hat die Arbeitsgruppe im August 2014 in Bern eine Tagung durchgeführt, in deren Rahmen die Anliegen der Stakeholder gesammelt und gebündelt wurden. Daraus entstand schliesslich eine finalisierte Fassung, die vom SAMW-Vorstand an seiner Sitzung vom 3. November 2014 diskutiert und definitiv verabschiedet wurde. 
ist der Einsatz von Informations- und Kommunikationstechnologie (eHealth) unerlässlich. Eine geeignete Infrastruktur trägt dazu bei, informelle Begegnungen zwischen den Berufsgruppen und eine transparente Kommunikation zu ermöglichen

\section{Die Leitungsstrukturen orientieren sich an inhaltlichen Zielen.}

Gesundheitseinrichtungen legen Leitungsstrukturen nach sachlichen Aspekten und nicht aufgrund von Statusüberlegungen fest und unterstützen damit das berufsübergreifende Arbeiten wirkungsvoll. Für das Wahrnehmen von Leitungsfunktionen in interdisziplinären Teams bedarf es der entsprechenden Kompetenz.

\section{Für alle Berufsgruppen sind die Qualitäts- sicherung und die Evaluation des Nutzens für den Patienten und die Gesellschaft ein Teil ihrer professionellen Haltung.}

Die Einführung und Etablierung der Zusammenarbeit zwischen allen Gesundheitsberufen ist mit geeigneter Versorgungsforschung zu begleiten.

\section{Umsetzung}

- Berufs- und Fachverbände, Gesundheitsinstitutionen (z. B. Spitäler, Netzwerke, Spitexorganisationen, stationäre Langzeitpflege) und Bildungsinstitutionen des Gesundheitsbereichs lancieren in ihren Gremien eine vertiefte Diskussion über die Charta und deren Bedeutung für die eigene Organisation.

- Die Berufs- und Fachverbände starten Pilotprojekte zur Beschreibung von Kompetenzen und Verantwortlichkeiten in konkreten Arbeitssituationen, zur Ausarbeitung von interprofessionellen Leitlinien und zur Organisation gemeinsamer Fortbildungsveranstaltungen.

- Die Berufsverbände setzen sich gemeinsam für die als notwendig erkannten Anpassungen von Gesetzen, Verordnungen und Tarifen ein.

- Die Charta dient als Lehrmittel und Diskussionsbasis in der Aus-, Weiter- und Fortbildung und als Stimulus für die gemeinsame Entwicklung von Lehrplänen.

- Die Charta bildet die Basis für den institutionalisierten Kontakt der Verbände und Ausbildungsstätten.

- Die SAMW begleitet den Prozess der Weiterentwicklung der Charta: Sie erstellt - im Sinne eines Monitorings - eine Übersicht über damit verbundene Initiativen und Projekte und organisiert spätestens zwei Jahre nach Veröffentlichung der Charta ein Treffen der Stakeholder, in deren Rahmen a) Erfahrungen ausgetauscht werden, b) die Charta gegebenenfalls angepasst wird und c) ein «Award» für besonders gelungene Zusammenarbeitsprojekte verliehen wird.

\section{Literatur}

- American College of Physicians (ACP). The Advanced Medical Home: A Patient-Centered, Physician-Guided Model of Health Care; 2006.

- World Health Professions Alliance (WHPA). Joint Health Professions Statement on Task Shifting; 2008.

- World Health Organisation (WHO). Task shifting: rational redistribution of tasks among health workforce teams; 2008.

- American College of Physicians (ACP). Nurse Practitioners in Primary Care; 2009.

- Frenk J et al. Health Professionals for a new century: transforming education to strengthen health systems in an interdependent world. Lancet. Report; 2010. (deutsche Übersetzung unter www.careum.ch/ lancet-report)

- WHO. Framework for action on interprofessional education and collaborative practice. Geneva; 2010.

- Robert-Bosch-Stiftung: Memorandum «Kooperation der Gesundheitsberufe»; 2011.

- Schweizerische Akademie der Medizinischen Wissenschaften (SAMW). Die zukünftigen Berufsbilder von Ärztinnen und Pflegenden - Bericht und Kommentar; 2011.

- Arbeitsgruppe BAG/GDK: Neue Versorgungsmodelle für die medizinische Grundversorgung; 2012.

- Witte F. Mehr kooperieren statt delegieren. Schweiz Ärztezeitung. 2012;93(22):809-11.

- American Medical Association. The structure and functioning of Interprofessional health care teams. Joint report of the Council of Medical Education and the Council Medical Service. Chicago; 2012.

- Ono T,Lafortune G, Schoenstein M. Health Workforce Planning in OECD Countries: a Review of 26 Projection Models from 18 Countries. OECD Health Working Paper No. 62; 2013.

- Plattform «Zukunft Ärztliche Bildung». Bericht «Interprofessionalität»; 2013.

- Donelan K. et al. Perspectives of Physicians and Nurse Pratitioners on Primary Care Practice. NEJM. 2013;368:1898-1906.

- Iglehart J. Expanding the Role of Advanced Nurse Practitioners - Risk and Rewards. NEJM. 2013;368:1935-41.

- APPG. All the Talents. How new roles and better teamwork can release potential and improve health services. London: All-Party Parliamentary; 2013.

- Careum. Neuausrichtung der Ausbildung für Gesundheitsberufe. Zürich; 2013.

- Sottas B et al. Umrisse einer neuen Gesundheitsbildungspolitik. Zürich: Careum Verlag; 2013

- European Interprofessional Practice and Education Network (EIPEN). Charter für Interprofessional Practice and Education; 2014

- Robert-Bosch-Stiftung. Gesundheitsberufe neu denken. Stuttgart; 2014

- Walkenhorst U, Mahler C, et al. Interprofessionelle Ausbildung in den Gesundheitsberufen. Positionspapier der Gesellschaft für medizinische Ausbildung (GMA). Erlangen; 2014. 